\title{
Phenformin in Insulin-dependent Diabetics
}

\author{
ARNOLD BLOOM,* M.D., F.R.C.P. ; R. J. KOLBE, $†$ M.B., M.R.C.P.
}

\begin{abstract}
Cummary: Forty-one diabetic patients on insulin $\checkmark$ were given $100 \mathrm{mg}$. of phenformin daily for six weeks, either before or after a period of six weeks of inert capsules, in a double-blind cross-over trial. Eleven patients while on phenformin noticed hypoglycaemic effects and reduced their insulin on average by almost $20 \%$ without resultant rise in blood sugar levels. Twenty-eight patients felt no untoward effects and maintained their usual insulin dose. Phenformin led to improved control of the diabetes, with a significant decrease in blood sugar levels and a significant reduction in the variability of the weekly blood sugar readings. There was no increased ketosis, no change in cholesterol, and no significant loss of weight.
\end{abstract}

\section{Introduction}

The amount of insulin a diabetic injects is a compromise between two conflicting objectives. On the one hand, the blood sugar should be maintained at as little above normal as possible. On the other hand, hypoglycaemic reactions must be avoided. Since blood sugar levels tend to fluctuate considerably in most insulin-dependent diabetics, the levels obtained in practice are much above normal during most of the day. Attempts to lower these levels by increasing the insulin dosage may lead to hypoglycaemic reaction. Indeed, as Somogyi (1959) pointed out, depression of the blood sugar by insulin provokes a reactive hyperglycaemia, so that successive increases of insulin dosage may not materially affect the ultimate blood sugar levels. Consequently, physicians experienced in the care of diabetic patients have come to realize the limitations of insulin therapy and to accept that many patients maintain blood sugar levels greatly in excess of normal. Though it is unlikely that hyperglycaemia is the sole cause of the degenerative changes that occur in long-standing diabetes, the consensus of evidence is that hyperglycaemia is an important contributory factor (Bloom, 1967). Hence any form of therapy that successfully reduces the blood sugar without leading to hypoglycaemic reactions must merit serious consideration.

More than 12 years' clinical experience has now accrued in the use of phenformin in diabetes, and its value has been established in maturity-onset diabetics not requiring insulin. Its mode of action has not been fully elucidated but, unlike the sulphonylureas, it can lower blood sugar levels in the absence of a functioning pancreas. Phenformin has been shown to reduce insulin requirements in labile diabetics (Pomeranze et al., 1957; Krall, 1959; Stowers, 1960), but no critical evaluation has been undertaken of the effect of phenformin in insulindependent diabetics in general.

The object of this study was to establish whether a welltolerated dose of phenformin given to insulin-dependent diabetics could successfully reduce blood sugar levels without increasing hypoglycaemic reactions.

\section{Method and Trial}

A double-blind cross-over trial using phenformin or inert capsules twice a day for 12 weeks was conducted in 47 insulin-dependent diabetics attending the diabetic clinic. Patients were asked if they were willing and able to attend regularly

* Physician, Whittington Hospital, London N.19

†Senior House-Physician, Whittington Hospital, London N.19 each week at a special session. It was explained that the trial was designed to see if the capsules would improve control of the diabetes. Patients were randomly allocated phenformin or dummy capsules during the first six weeks and then dummy or phenformin capsules for a further six weeks. Thus all patients had six consecutive weeks on phenformin capsules $50 \mathrm{mg}$. twice a day, some in the first six weeks, some in the second six weeks. Neither the patients nor the clinician in charge (R.J.K.) were aware of the nature of the capsules currently allocated.

The phenformin capsules were of the slow-release type in which granules of phenformin were mixed with shellac. The inert capsules were identical in appearance and composition with the active capsules except that they did not contain phenformin. Capsules were supplied in containers sufficient to last for nine days. Since the patients attended each week this allowed a check to be made on the number of capsules left in the bottle. Each bottle was numbered and lettered to correspond with the patient's number and the letter of the week of the trial, the random allocation of numbers having been handled independently of the clinician in charge. Patients were seen each week at the same time of the day, usually within a few minutes of previous attendances. In a few instances where the patient was unable to attend at a particular clinic, arrangements were made for the appropriate bottle of capsules to be supplied.

The 47 patients ( 26 male and 21 female) were aged 12 to 73 years, mean 39. The mean duration of the diabetes was 129 months (range 14 to 420 ). The mean insulin requirement daily was 52.7 units (range 10 to 104). Fourteen patients were on insulin twice a day and 33 on a single daily injection, 23 being on a mixture of soluble and zinc protamine insulin and 10 on isophane insulin or insulin zinc suspension either alone or mixed with soluble insulin. Blood sugars were estimated on venous blood with the automated guaiacum glucose oxidase method (Gutteridge and Wright, 1968). Serum cholesterol was measured by the auto-Technicon methodology as modified by Levine and Zac (1964). The weight in clothes was recorded and a freshly passed urine specimen tested for sugar (Clinitest) and ketones (Acetest). A general inquiry was made at each visit for symptoms, and direct questions were asked about hypoglycaemic reactions, nausea, loss of appetite, and bowel upsets. Advice concerning insulin dosage was not proffered, but changes in insulin dosage made by patients were recorded.

\section{Results}

Six patients did not complete the trial. One forgot to take the capsules with him on holiday. Two patients stopped taking the capsules, one because she developed salpingitis and one because he felt weak and ill; the capsules in these two cases were inert. Three others stopped taking the capsules, and these proved to be phenformin-one developed gastroenteritis after four weeks, one developed an influenza-like illness with nausea after two weeks, and one developed troublesome hypoglycaemic reactions. The other 41 patients completed the trial, the results being available for analysis.

In response to direct questioning seven patients noted transient nausea during the first few weeks of phenformin, but this did not persist, two experienced nausea both on the dummy capsules and on phenformin, and three noted nausea on the dummy capsules but not on phenformin. No patient complained of diarrhoea. 
Thirteen patients altered their insulin dosage during the trial and were analysed separately from the 28 who maintained the same insulin dosage throughout. It should be remembered that most of the patients in the trial had been taking insulin for many years, and some were in the habit of adjusting the dosage from time to time according to the response.

\section{Patients Who Maintained Same Insulin Dosage}

Hypoglycaemia.-In this group hypoglycaemic reactions were non-existent or mild. In response to direct questioning seven patients noted slight reactions from time to time, occurring both when taking phenformin and when taking inert capsules.

Blood Sugar Levels. - The actual blood sugar readings of the patients attending are recorded in the Table. In patients who started on inert capsules the blood sugar levels fell when they were transferred to phenformin (see Chart). In those who started on phenformin the levels rose when they were transferred to dummy capsules. The mean level while taking dummy capsules (geometric mean) was $164 \mathrm{mg} / 100 \mathrm{ml}$. as compared with $121 \mathrm{mg} . / 100 \mathrm{ml}$. while taking phenformin. Thus phenformin reduced the blood sugar level on average by $26.2 \%$, and a $t$ test in an analysis of variance (of log blood sugar level) showed this decrease to be highly significant $(\mathrm{P}<0.001 ; t=4.57$ with 306 degrees of freedom). The Chart also shows that the effect of phenformin was already apparent at the end of the first week, and that there was no evidence of any carry-over of the effect at the end of the first week of dummy treatment. The variability of the weekly blood sugar readings was reduced during phenformin treatment compared with the period of dummy treatment. The standard deviation of the weekly readings on individual patients was 63.9 during phenformin treatment and 80.0 during the dummy treatment. The reduction, by $20.1 \%$, is highly significant $(P<0.001$ in a variance ratio test). The decrease in blood sugar during phenformin treatment appeared to be independent of the blood sugar level of the individual. Moreover, the decrease for the patients on a mixture of soluble and protamine zinc insulin once daily was similar to that for those on other forms of insulin.

Urinary Sugar and Ketones.-During phenformin treatment more urines were recorded with $0.25 \%$ or no sugar and fewer

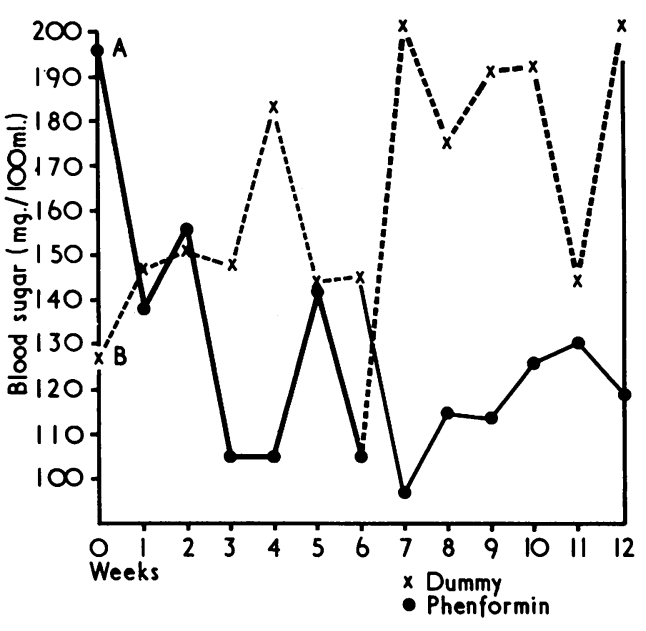

Average weekly blood sugar levels in 28 patients on unaltered insulin dosage while on phenformin or dummy capsules. Geometric means. (A) First six weeks on phenformin, followed by six weeks on dummy (14 patients). (B) First six weeks on dummy, followed by six weeks on phenformin (14 patients).

with $2 \%$ or more sugar than when taking inert capsules. These findings reflected the changes in blood sugar readings. Acetone was seldom present in the urine, and was found with no greater frequency during phenformin therapy than when inert capsules were taken.

Weight.-There were no significant changes in the weight of patients during the trial. The mean increase was $0.4 \mathrm{lb}$. (180 g.) during the period of inert capsules. During the phenformin period the mean weight loss was $0.04 \mathrm{lb}$. $(18 \mathrm{~g}$.) in the group whose insulin remained unchanged.

\section{Patients Who Altered Their Insulin Dosage}

Hypoglycaemia.-Thirteen patients reduced their insulin of their own volition because of hypoglycaemic reactions, all but two of them while taking phenformin. In four of these patients on phenformin the reactions were troublesome and in the remainder they were mild.

Blood Sugar Readings on Patients Taking Capsules, Insulin Dosage Unchanged. Figures in Black After Phenformin. Figures in Italic After Inert Capsules or at First Attendance

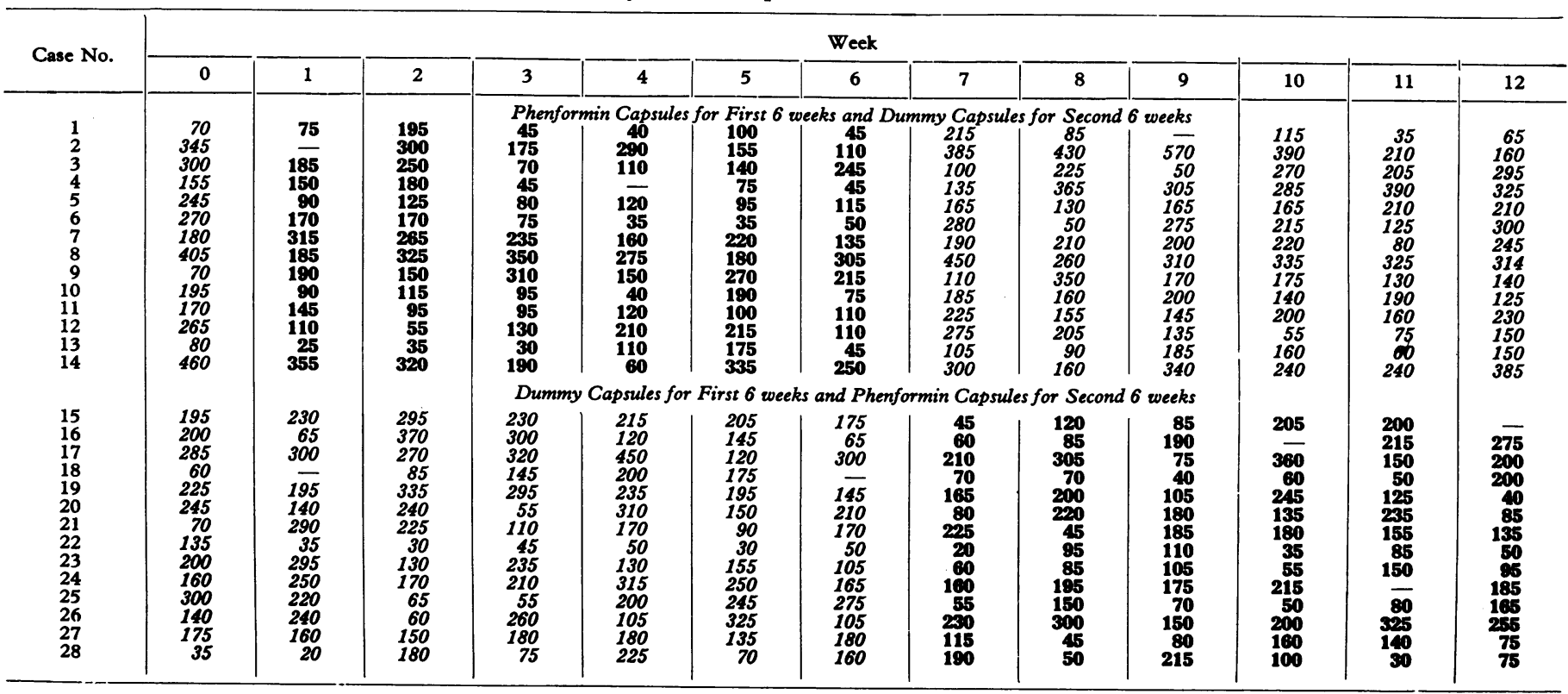


Changes in Insulin.-Six patients lowered the dose of insulin while taking phenformin during the first half of the trial and four of these raised the dose again when transferred to the inert capsules. Five had maintained the same insulin dose during the initial dummy period but reduced their insulin when transferred to phenformin. Two lowered their insulin while on the inert capsules and remained on the lower dose when changed to phenformin. The mean initial daily dose in the eleven patients on phenformin who reduced their insulin was $\mathbf{5 0 . 4}$ units, falling to $\mathbf{4 0 . 0}$ units at the end of the phenformin period-a reduction of $19.8 \%$.

Blood Sugar Levels. - The geometric mean blood sugar in patients in this group on inert capsules was $136 \mathrm{mg} . / 100 \mathrm{ml}$., compared with $116 \mathrm{mg} . / 100 \mathrm{ml}$. during phenformin therapy, a reduction of blood sugar on average of $8.5 \%$, not statistically significant.

\section{Cholesterol}

No important change was observed in the cholesterol levels of the 38 patients for whom the information was available. The mean level before phenformin was $203.8 \mathrm{mg} . / 100 \mathrm{ml}$. (range 260 to 145) and after phenformin $204.7 \mathrm{mg} . / 100 \mathrm{ml}$. (range 275 to 165 ).

\section{Discussion}

Phenformin has been used in insulin-dependent diabetics where excessive lability of blood sugar levels occurs (inappropriately named "brittle diabetics"), in the hope that replacement of some of the insulin by phenformin would lead to improved stability. There has been a lack of enthusiasm for this manoeuvre on at least two counts: firstly, because there is a high incidence of side-effects when phenformin is given in doses large enough to be effective (Oakley, 1968), and, secondly, because life is complicated enough for the brittle diabetic without the added trouble of taking phenformin (Malins, 1968). The present trial does not suggest that these premises are valid when phenformin is administered in capsule form at a dose level of $100 \mathrm{mg}$. daily. Patients appeared unaware when a dummy capsule was substituted for phenformin, or when phenformin was substituted for the dummy. No patient complained of difficulty in taking the capsules or suggested that the routine was troublesome.

Gastrointestinal effects were minimal. In only two out of 47 patients did the capsules have to be discontinued because of gastrointestinal upset, and in neither case was it certain that the effect was due to phenformin rather than intercurrent infection. In nine patients who on dirett questioning complained of nausea the symptom was transient; similar symptoms were experienced by five patients on dummy capsules. On direct questioning most patients made no complaint of any gastrointestinal disorder.

Eleven out of 41 patients taking phenformin developed hypoglycaemic symptoms and for this reason reduced their insulin. The mean reduction of insulin in these cases was nearly $20 \%$. Despite this considerable reduction the blood sugar levels when taking phenformin were still marginally (though not significantly) lower than when on the higher insulin dose. In patients liable to hypoglycaemic reactions it would seem prudent initially to reduce the insulin dose with the introduction of phenformin. The dose of insulin could then be later increased if control was still not satisfactory.

In 28 out of 41 patients taking phenformin hypoglycaemic reactions were unexceptionable and the insulin dose was left unaltered. In this group the blood sugar levels were reduced by more than $25 \%$ and the variability of the week-by-week blood sugar readings was reduced by $20 \%$. In other words, the addition of two 50-mg. phenformin capsules successfully lowered the blood sugar levels in these insulin-dependent diabetics without causing hypoglycaemic reactions. At the same time the blood sugar fluctuations were greatly diminished. If it is accepted that hyperglycaemia and rapidly changing blood sugar levels are both ultimately harmful, this therapy could offer material advantages in diabetics difficult to control well on insulin alone.

Many investigators have shown that phenformin reduces the cholesterol level in maturity-onset diabetics (Alterman and Lopez-Gomez, 1968; Gershberg et al., 1968; Schaefer, 1968), but the effect is most pronounced where the levels of cholesterol are markedly raised. Indeed, Gershberg et al. found no change in patients with an initial cholesterol level below 250 mg. $/ 100 \mathrm{ml}$. Only two patients in the present series exceeded this initial value (one 260 and one $255 \mathrm{mg} . / 100 \mathrm{ml}$.), so the fact that phenformin caused no fall in cholesterol levels in patients in this trial is consistent with the findings of these observers. It has been generally assumed (Harsant, 1969) that the decrease in cholesterol is an indirect result of the reduction in serum insulin levels produced by phenformin in maturity-onset diabetics. If this were so, a fall in cholesterol would not be expected in insulin-dependent diabetics who cannot produce endogenous insulin.

Phenformin leads to a loss of weight in overweight maturityonset diabetics (Patel and Stowers, 1964; Schwartz et al., 1966; Mirsky, 1968), but in usual therapeutic doses no change can be detected in obese non-diabetics (Baird, 1969). In this study, though blood sugars were considerably reduced, no important weight change occurred.

There was no evidence of increased ketosis due to phenformin on regular urine examinations. In keto-acidoticprone diabetics not taking insulin, phenformin leads to ketosis (Hall et al., 1958), probably because in insulin-deficient states the liver produces excess ketones (as well as glucose), and this effect is not suppressed by phenformin. If sufficient exogenous insulin is administered, however, ketosis is inhibited and the administration of phenformin is therefore irrelevant in this respect.

We express our gratitade to: Dr. Ian Sutherland, of the M.R.C. Statistical Research and Services Unit, University College Hospital Medical School, for help in the preparation of the trial and the statistical interpretation of the results; Dr. J. B. Spooner and Mr. P. R. Harsant, of Bayer Products Company, Surbiton, for the supplies of capsules; Dr. T. Dormandy and the biochemistry department, Whittington Hospital, for the biochemical results; and the North-West Metropolitan Regional Hospital Board for a grant to pursue these studies.

\section{REFERENCES}

Alterman, S. L., and Lopez-Gomez, A. A. (1968). Annals of the New York Academy of Sciences, 148, 884.

Baird, M. (1969). Postgraduate Medical fournal, 45, May Suppl., p. 17. Bloom, A. (1967). Proceedings of the Royal Society of Medicine, 60, 149. New Yor, Javier, Z., Hulse, M., and Hecht, A. (1968). Annals of the New York Academy of Sciences, 148, 914

Wright, E. B. (1968). Fournal of Medical Laboratory Technology, 25, 385

Hall, G. H., Crowley, M. F., and Bloom, A. (1958). British Medical fournal, 2, 71.

p. 20. Krall, L. P. (1959). Annals of the New York Academy of Sciences, 82, 603.

Levine, J. B., and Zak, B. (1964). Clinica Chimica Acta, 10, 381.

Malins, J. (1968). Clinical Diabetes Mellitus, p. 376. London Eyre and Spottiswoode.

937. S. (1968). Annals of the New York Academy of Sciences, 148,

Patel, D. P., and Stowers, J. M. (1964). Lancet, 2, 282.

the Society for Experimental Mouratoff, G. T. (1957). Proceedings of

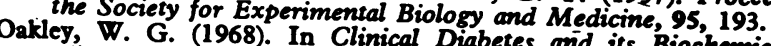

edited by W. B . In Clinical Diabetes and its Biochemical Basis, Oxford, Blackwell Scientific.

Schaefer, L. E. (1968). Annals of the New York 148, 925.

Schwartz, M. J., Mirsty, S. and Schacfer, I B (1966). Metabolism, 808.

Somogyi, M. (1959). American Fourmal of Medicine, 26, 169.

603. J. (1960). Proceedings of the Royal Society of Medicine, 53, 